\title{
AN INEQUALITY FOR LINEAR TRANSFORMATIONS WITH EIGENVALUES
}

\author{
BY C. A. SWANSON
}

Communicated by R. P. Boas, September 14, 1961

The purpose of this announcement is to state theorems concerning bounded linear transformations on Hilbert space which are far more general than the recent theorems of H. D. Block and W. H. J. Fuchs [2]. Our theorems are more general even in the case that the transformation is a matrix, as in [2]. The basic idea involved in these theorems was first communicated to the author by Professor H. F. Bohnenblust in 1957, and has been applied meanwhile to various perturbation problems for ordinary and partial differential equations, $[1 ; 4]$. The theorems in essentially their present form were enunciated by the author in an unpublished manuscript sent to Professor Bohnenblust in July, 1960.

THEOREM 1. Let $T$ be a bounded linear transformation on Hilbert space $\mathfrak{S}$ having a complete orthonormal set of eigenelements $y_{i}$. Let $\alpha$ be an arbitrary complex number and let $\epsilon$ be a nonnegative real number. Let $P(\epsilon)$ be the projection operator from $\mathfrak{S}$ onto the subspace $\mathfrak{F}(\epsilon)$ spanned by all the $y_{i}$ whose corresponding eigenvalues $\lambda_{i}$ lie in the open disk $|\lambda-\alpha|<\epsilon$. Then for any $x \in \mathfrak{S}$,

$$
\|T x-\alpha x\| \geqq \epsilon\|x-P(\epsilon) x\| .
$$

Proof. By hypothesis, $T y_{i}=\lambda_{i} y_{i}$ and $\left(y_{i}, y_{j}\right)=\delta_{i j}$. It is easily verified that $\left(T x-\alpha x, y_{i}\right)=\left(\lambda_{i}-\alpha\right)\left(x, y_{i}\right)$, and hence the Parseval completeness formula gives

$$
\|T x-\alpha x\|^{2}=\sum_{i}\left|\lambda_{i}-\alpha\right|^{2}\left|\left(x, y_{i}\right)\right|^{2} \geqq \epsilon^{2} \sum_{i}^{*}\left|\left(x, y_{i}\right)\right|^{2},
$$

where the ${ }^{*}$ denotes summation over only those indices $i$ for which $\left|\lambda_{i}-\alpha\right| \geqq \epsilon$. The result (1) is then immediate.

The following theorem is a corollary of Theorem 1.

THEOREM 2. Let $T$ be a symmetric, completely continuous linear transformation on Hilbert space $\mathfrak{S}$. Let $\alpha$ and $\epsilon$ be real numbers with $\epsilon$ nonnegative. Let $P(\epsilon)$ be the projection operator onto the subspace $\mathfrak{F}(\epsilon)$ spanned by all the eigenelements of $T$ whose corresponding eigenvalues lie in the interval $(\alpha-\epsilon, \alpha+\epsilon)$. Then for any $x \in \mathfrak{S}$, the inequality (1) is valid. 
Theorem 1 implies many other results, among them the theorems of Block and Fuchs [2]. The latter are obtained if $T$ is a matrix acting on finite dimensional Euclidean space and the choices

$$
\alpha=(T x, x) ; \quad \epsilon=\min _{i}\left|\lambda_{i}-\alpha\right| ; \text { and }\|x\|=1
$$

are made. In this case, one verifies that $\|T x-\alpha x\|^{2}=\|T x\|^{2}-|\alpha|^{2}$, and hence (1) reduces to

$$
\min _{i}\left|\lambda_{i}-\alpha\right| \leqq\left(\|T x\|^{2}-|\alpha|^{2}\right)^{1 / 2} .
$$

The main application envisaged by the author is to perturbation theory for ordinary and partial differential operators when the perturbation arises from a change of the domain of the operator $[1 ; 3 ; 4]$. In this case $\mathfrak{E}$ is taken to be the Lebesgue space $L^{2}(E)$, where $E$ is an open, connected region in Euclidean or Riemannian space. One finds in such applications that there is an $m$-dimensional subspace $\mathfrak{D}$ of $\mathfrak{S}$ such that

$$
\|T x-\alpha x\| \leqq \delta\|x\|
$$

for all $x \in \mathfrak{D}$, and some numbers $\alpha, \delta$ with $\delta$ nonnegative. One concludes from (1) and (2) that $\|x-P(2 \delta) x\| \leqq 1 / 2\|x\|$ for all $x \in \mathfrak{D}$. Then $P(2 \delta) x=0$ implies $x=0$. Hence $\operatorname{dim} \mathfrak{F}(2 \delta) \geqq \operatorname{dim} \mathfrak{D}$ and at least $m$ eigenvalues $\lambda_{i}$ of $T$ lie in the disk $\left|\lambda_{i}-\alpha\right|<2 \delta$. In the applications, one usually has additional information from which one can deduce that there are exactly $m$ eigenvalues in this disk. Also, various properties of eigenelements can be obtained by these methods.

\section{REFERENCES}

1. H. F. Bohnenblust, C. R. DePrima and C. A. Swanson, Elliptic operators with perturbed domains, to appear.

2. H. D. Block and W. H. J. Fuchs, An enclosure theorem for eigenvalues, Bull. Amer. Math. Soc. vol. 67 (1961), pp. 425-426.

3. C. A. Swanson, Differential operators with perturbed domains, J. Rational Mech. Anal. vol. 6 (1957), pp. 823-846.

4. —-, Asymptotic estimates for limit circle problems, Pacific J. Math., to appear.

The University of British Columbia 